\title{
Coevaluación como vía de aprendizaje: dos experiencias en el Grado de Economía de la USC
}

\author{
Co-assessment as a learning path: two experiences in the Degree of \\ Economic at the USC
}

\section{Eva Aguayo Lorenzo*, Nélida Lamelas Castellanos**, Ana Iglesias Casal*, Carmen López Andión*, Xosé Antón Rodríguez González*}

\author{
* Departamento de Economía Cuantitativa \\ **Departamento de Economía Aplicada \\ Fac. CC. Económicas y Empresariales \\ Universidade de Santiago de Compostela \\ eva.aguayo@usc.es nelida.lamelas@usc.es ana.iglesias.casal@usc.es \\ carmen.lopez.andion@usc.es xoseanton.rodriguez@usc.es
}

\begin{abstract}
Resumen
En este trabajo presentamos y compartimos las experiencias de la utilización de la coevaluación como metodología docente aplicada en clases interactivas de las asignaturas Econometría II y Política Económica I del Grado en Economía de la Universidade de Santiago de Compostela, durante el curso 2011-12. En ambas experiencias, se confirma la contribución de esta estrategia de evaluación en la formación en competencias y en el proceso de aprendizaje autónomo del alumnado.
\end{abstract}

Palabras clave: Coevaluación, Enseñanza-aprendizaje, Educación superior.

\begin{abstract}
In this paper we present and share the experiences in the use of co-assessment as a teaching methodology in the interactive classes of the Econometrics II and Economic Policy I courses, which belong to the Degree of Economics at the University of Santiago de Compostela. In both experiences, carried out during the 2011-12 academic year, this type of evaluation strategy confirmed its positive contribution to the students' autonomous learning process and, above all, to the development of some cognitive and procedural competences.
\end{abstract}

Key words: Co-assessment, Teaching-Learning, Higher Education.

\section{Introducción}

En el actual Espacio Europeo de Educación Superior, la formación en competencias (Zambrana, 2010) y el aprendizaje autónomo del alumnado (Sánchez, 2010) constituyen objetivos básicos. En este contexto, cobra especial relevancia la modificación de los criterios y estrategias metodológicas de evaluación (Gessa, 2011; Gómez y Quesada, 2017) así como la coevaluación o evaluación compartida en la que el profesorado comparte la responsabilidad de la evaluación con el alumnado.

De esta forma, el rasgo distintivo de la coevaluación es involucrar de forma activa al estudiantado en la toma de decisiones en la tarea de evaluación (Somervell, 1993; Topping, 1998). Asimismo, mediante esta participación, se consigue una opinión más realista de sus propias capacidades ya que pueden hacer juicios más racionales al evaluar los logros de sus compañeros de clase (Dochy et al., 2002) a la vez que también les permite la reflexión, aumentar sus metas de aprendizaje y, como resultado de este desafío, 
aprender más y mejor (Falshikov, 1986; Hacker et al., 2000). En definitiva, siguiendo a Hall (1995), el proceso de coevaluación proporciona la posibilidad de que el alumnado comparta el rol del docente en la tarea de evaluación y desarrolle habilidades de autoevaluación asociadas a esta función, sin la pérdida por parte del profesorado del control sobre la evaluación final (Dochy et al., 1999; Álvarez-Valdivia, 2008).

La evaluación interpares, por los criterios relevantes que aporta acerca del trabajo de sus colegas, (Falchikov, 2001); puede resultar un complemento muy útil a la evaluación del profesorado en cualquiera de sus distintas categorías: intragrupo, intergrupo o individual, (Prins et al., 2005). Son precisamente estas dos últimas las que se aplicarán en las experiencias realizadas en nuestras materias.

Entre sus principales ventajas generales apreciamos su contribución al conocimiento de los contenidos y a la solución de problemas, el desarrollo de debates y valoraciones críticas de diferentes temáticas y el respeto a la diversidad de opiniones. Particularmente, la evaluación intergrupo puede constituir una importante herramienta que dinamice y fortalezca el trabajo colaborativo, en tanto que la evaluación individual puede tener mayor incidencia en fomentar la capacidad de argumentación y las relaciones interpersonales.

Además, la combinación de la evaluación entre iguales y la evaluación realizada por el profesorado otorga un valor añadido a la coevaluación, mejorando la calidad del aprendizaje. Este proceso de coevaluación involucra activamente al estudiantado en la tarea de evaluación compartiendo la responsabilidad y los criterios de calificación con el profesorado, y clarificando los objetivos del aprendizaje.

\section{Dos experiencias de coevaluación en el Grado en Economía}

Las experiencias que a continuación presentamos se desarrollaron en las asignaturas Econometría II y Política Económica I, durante el curso 2011-12. Ambas son materias obligatorias pertenecientes al tercer curso del Grado en Economía de la Universidade de Santiago de Compostela (USC, 2009).

El diseño de ambas actividades se ha basado en una metodología de trabajo que no sólo propicia la participación del estudiantado en la evaluación sino que, al hacer posible que los resultados de la misma retornen a éste, retroalimenta competencias cognitivas y procedimentales ya adquiridas. En cuanto a las competencias relacionadas con los conocimientos propios de cada materia, éstos se ven reforzados debido a que para poder evaluar determinados aspectos de un contenido es necesario previamente profundizar en éstos. A la vez, ello también sucede cuando se reciben valoraciones que aportan explicaciones sobre la temática objeto de estudio. Respecto a las competencias procedimentales, se potencia el ejercicio de la crítica y la autocrítica y las capacidades relacionadas con el trabajo grupal, entre otras.

En el primer caso la evaluación del profesorado es complementada con la de evaluación entre grupos y en el segundo caso con la evaluación individual entre estudiantes.

\subsection{Evaluación compartida en la materia Econometría II}

\section{Actividad propuesta}

Se plantea la resolución de un caso econométrico práctico, en grupos de tres estudiantes y de forma colaborativa. El planteamiento de la resolución de casos prácticos permite incidir en el aprendizaje autónomo del alumnado (Aguayo, 2010). La plataforma virtual sirve de soporte para todos materiales 
(enunciado del ejercicio, fichero de datos, pautas para la realización del trabajo,..) que deben utilizar a lo largo de la actividad.

\section{Metodología y desarrollo del trabajo}

En una primera clase interactiva, cada grupo dispone de un ordenador para resolver el caso práctico. El profesorado resuelve las dudas que puedan surgir y supervisa la interacción en cada grupo.

En una segunda clase práctica cada grupo entrega en papel el caso resuelto. Tras su recogida, el profesorado reparte aleatoriamente los trabajos para realizar una corrección interpares mediante la evaluación entre grupos. Cada grupo debe cubrir un formulario con las valoraciones que le asigna a distintos aspectos tales como presentación formal y estructura, claridad expositiva, interpretación correcta y resultados obtenidos. La utilización de rúbricas permite tener una información detallada de los aspectos que se van a tener en cuenta y las valoraciones asignadas por nivel de consecución. Uno de los objetivos de la actividad es que el alumnado no sólo sepa realizar un informe econométrico, sino que también desarrolle destrezas para su valoración y evaluación.

La evaluación final de la actividad comprende la nota asignada por el grupo corrector y la puntuación asignada por profesorado. La evaluación realizada por el grupo de estudiantes tiene un peso de un $30 \%$ en la nota final, mientras que el $70 \%$ corresponde a la evaluación del profesorado. Esta evaluación efectuada por el profesorado se desglosa en un $60 \%$ asignado a la corrección del caso práctico y un $10 \%$ a la valoración de la capacidad correctora.

\section{Resultados}

Los resultados de esta actividad han sido muy positivos. Desde el punto de vista del profesorado implicado, cabe destacar el aumento de la participación en clase, la interacción entre el alumnado y la dinámica de trabajo en equipo, generadas en la corrección del caso práctico de otro grupo. Para conocer la opinión del alumnado implicado (64 estudiantes) se decidió evaluar la metodología utilizada mediante un cuestionario anónimo. Dicho cuestionario consistía en diez preguntas cuyas respuestas estaban definidas mediante una escala ${ }^{1}$ de 5 puntos. En la Tabla 1 recogemos las respuestas de todo el alumnado a los ítems relacionados con la evaluación interpares.

El aspecto menos valorado ha sido el grado de implicación en la asignatura que conlleva la corrección interpares (pregunta 3), con una puntuación en torno a 3,43. Mientras que entre los más valorados, con puntuaciones ligeramente superiores al 4, se encuentran darse cuenta de aspectos que no habían detectado (pregunta 1), aumentar el grado de implicación en la corrección del ejercicio (pregunta 5) y la mejora en la capacidad autoevaluadora (pregunta 6).

Entre los comentarios, realizados por el alumnado, podemos señalar:

"Esta actividad es muy positiva, dado que nos ayuda a ser críticos con uno mismo y con los demás".

"Creo que no es del todo justo que nos evaluemos los unos a los otros, dado que aunque podemos conocer la materia y el trabajo, no creo que debamos detentar el poder de decisión de la nota que merecen nuestros compañeros, creo que es una tarea que corresponde a la profesora".

“...al corregir el trabajo de otro grupo me ayudó a darme cuenta de aspectos que no habíamos puesto, mejoré mi capacidad de autoevaluación".

\footnotetext{
${ }^{1}$ Las medidas de satisfacción e importancia se representan con una valoración que va desde 1 (nada de acuerdo/muy mal) hasta 5 (totalmente de acuerdo/muy bien).
} 
RIDU Revista d'lnnovació Docent Universitaria $10(2018)$ 25-32

“...ha sido una buena actividad y entretenida que debería extenderse a otras asignaturas".

\begin{tabular}{|l|c|c|}
\hline & Media & \multicolumn{1}{|c|}{$\begin{array}{c}\text { Desviación } \\
\text { típica }\end{array}$} \\
\hline Participar en la corrección del caso práctico de otro grupo me ha ayudado a... \\
\hline $\begin{array}{l}\text { 1. Darme cuenta de aspectos que con mi grupo no habíamos } \\
\text { detectado }\end{array}$ & 4.02 & 1.31 \\
\hline 2. Mejorar mi capacidad crítica y de evaluación & 3.62 & 0.85 \\
\hline 3. Implicarme más en la asignatura & 3.43 & 0.95 \\
\hline $\begin{array}{l}\text { Que una parte de la nota de esta actividad sea la valoración que la profesora hace de nuestra } \\
\text { capacidad correctora me ha ayudado a... }\end{array}$ & 3.89 & 0.84 \\
\hline $\begin{array}{l}\text { 4. Reflexionar sobre la evaluación de un caso práctico y su } \\
\text { presentación }\end{array}$ & 4.05 & 0.83 \\
\hline $\begin{array}{l}\text { 5. Aumentar mi grado de implicación en la corrección del ejercicio } \\
\text { del otro grupo }\end{array}$ & 4.02 & 0.87 \\
\hline 6. Mejorar mi capacidad de autoevaluación
\end{tabular}

Tabla 1: Resultados del cuestionario de evaluación de la metodología de la actividad

\subsection{Evaluación compartida en la materia Política Económica I}

\section{Actividad propuesta}

Se plantea la exposición oral de los resultados de un trabajo de investigación realizado por el alumnado. Enmarcado en la temática general "Política económica y crisis actual" el alumnado debe elegir (según su preferencia) un subtema asociado a determinadas políticas económicas propuestas o implementadas en ese contexto.

Una vez realizado el trabajo de investigación, se expondrán oralmente los resultados alcanzados. Se solicita además la entrega de un breve informe con el título, importancia y objetivos del tema elegido, así como el índice de aquellos aspectos que tratará, sus conclusiones y la bibliografía consultada.

Para evitar duplicidades en los contenidos objeto de estudio, los subtemas se anotan en un listado, a medida en que van siendo seleccionados. Este listado queda a disposición del alumnado para su consulta. A efectos de garantizar la homogeneidad de las presentaciones, la profesora ofrece orientaciones acerca de su estructura y el tiempo disponible para la realización de éstas en clases interactivas.

\section{Metodología y desarrollo del trabajo}

Previo al inicio de la actividad, se seleccionaron aleatoriamente tres estudiantes que se encargaron de evaluar anónimamente cada exposición. Recibieron una ficha con los aspectos que debían tener en cuenta para la valoración y calificación. Éstos fueron:

- estructura y contenido de la presentación

- claridad expositiva

- originalidad y recursos o materiales utilizados

- respuestas a las preguntas formuladas. 
Se les solicitó además, que identificasen el aspecto que a su juicio resultaba más significativo de la exposición y la mayor limitación de la misma. La nota numérica se concedió en base a 10 puntos.

En cada clase interactiva se realizaron varias presentaciones. Con posterioridad a la exposición, se realizaron preguntas a cada ponente y se dio paso a un debate general. Al final del mismo se precisaron los aspectos más relevantes de las temáticas analizadas.

La profesora, incluyendo su propia evaluación, promedió las puntuaciones dadas por el alumnado ${ }^{2}$ y otorgó la nota. Las fichas con todos los señalamientos y evaluaciones se adjuntaron al informe que inicialmente había entregado cada estudiante Así, en una sesión posterior al concluir todas las exposiciones, cada ponente recibió su informe y pudo conocer todas las valoraciones anotadas por sus colegas y la profesora sobre el trabajo realizado.

\section{Resultados}

El alumnado se mostró muy interesado en participar en esta experiencia. En todos los grupos de clases interactivas en que se desarrolló esta técnica de evaluación reconocieron que les ofrecía la oportunidad como evaluadores y evaluadoras de expresar criterios sobre el trabajo de otros colegas y compararlos entre sí. A la vez, como receptores de la calificación, tuvieron la posibilidad de leer opiniones de diferentes personas (estudiantes y profesora) que en ocasiones resultaron coincidentes, pero que en otros casos manifestaron discrepancias en cuanto a las debilidades y fortalezas de su trabajo.

Los principales señalamientos realizados se agruparon por una parte, en torno a las capacidades desplegadas por el alumnado en su calidad de presentadores: la manera en que se expresaron, su interacción con el público, el uso de materiales audiovisuales; y por otra, en cuanto a los contenidos: la selección y el orden lógico de aspectos, el uso de datos para ejemplificar los diversos planteamientos, el hecho de ofrecer valoraciones propias sobre los problemas, entre otros.

Desde el punto de vista de la profesora la coevaluación resultó satisfactoria porque la labor de quienes evaluaron fue fundamentalmente justa y rigurosa. En ocasiones incluso su nivel de exigencia fue superior al esperado. Muy pocas valoraciones reflejaron calificaciones otorgadas sin un adecuado fundamento. Mayoritariamente, hubo coincidencia o complementariedad de criterios con la docente.

Las opiniones del alumnado sobre esta actuación fueron recogidas en las aulas, con una participación de más del $85 \%$ del total de la matrícula ${ }^{3}$ de la asignatura. Se les solicitó que escribieran de forma sintetizada sus criterios sobre la tarea, precisando aspectos positivos o satisfactorios y los negativos o limitaciones.

Un resumen de los argumentos planteados gira en torno a las siguientes apreciaciones: al tener que evaluar y puntuar el trabajo de otra persona, se presta mayor atención a las exposiciones y constituye un mayor incentivo para participar en el debate posterior sobre la temática. Al atender a contenidos interesantes que pueden incluir aspectos no tratados anteriormente en la materia, se facilita el proceso de apropiación de nuevos conocimientos o se establecen más fácilmente los vínculos con otros tópicos de la asignatura.

\footnotetext{
${ }^{2}$ Algunos autores consideran que se le debe asignar un porcentaje a la nota otorgada por el alumnado por ser menos fiable que la del profesorado. Sin embargo, se evidencia en la mayoría de los casos una correlación positiva entre las puntuaciones de estudiantes y docente con las calificaciones finales. Ibarra et al. (2012).

${ }^{3}$ Desde la creación del Grado en Economía el número de personas matriculadas en nuestra materia en cada curso oscila entre $80-110$.
} 
Además, realizar la evaluación y calificación de las exposiciones contribuye al desarrollo de la presentación propia dado que ayuda a detectar aspectos positivos y negativos que deben considerarse.

La tarea de dar nota resulta más difícil que la de hacer señalamientos; sobre todo por la inexperiencia en realizar la primera y la poca práctica en la segunda. También, ambas actuaciones aumentan la capacidad de crítica y autocrítica individual; así como la receptividad a las opiniones vertidas cuando éstas no provienen solamente de docentes, sino de iguales.

\subsection{Análisis comparativo de ambas experiencias}

En las dos asignaturas, la participación del alumnado en calidad de coevaluadores y coevaluadoras en el proceso de evaluar tareas docentes modificó la tradicional visión que considera al profesorado como único responsable de esta tarea. Las opiniones y las puntuaciones otorgadas por el estudiantado se tuvieron en cuenta para conformar la nota final del ejercicio realizado en cada caso.

En Econometría II, la asignación de un número a cada equipo permitió que se mantuviera el anonimato tanto en el desarrollo de la evaluación como en la recepción de los resultados. Mientras que en Política Económica I, al tratarse de una presentación oral, el anonimato sólo se refiere a la autoría de las valoraciones interpares recibidas en la fase final.

Las actividades propuestas requirieron de un cuidadoso trabajo preparatorio previo a las mismas por parte del profesorado. En este sentido destaca en la asignatura de Econometría II la formación de los grupos de trabajo, para la que se tuvo en cuenta la trayectoria de cada estudiante en la materia, a efectos de conformar equipos heterogéneos en su composición. En Política Económica I, la profesora realizó la asignación de responsables individuales de la evaluación atendiendo a que personas muy afines y acostumbradas a trabajar conjuntamente no se evaluaran entre sí.

En ambas actividades se alcanzó una alta participación y los resultados obtenidos fueron considerados satisfactorios tanto por el profesorado como por el alumnado.

\section{Conclusiones}

Algunas de las principales conclusiones son las siguientes:

En la actualidad el proceso de enseñanza-aprendizaje requiere del desarrollo de técnicas de trabajo que faciliten una participación cada vez más activa del alumnado. La conjunción de involucrar al estudiantado en las tareas de evaluación y por esa vía, encausar el retorno de sus resultados, contribuye a la obtención de competencias cognitivas y procedimentales necesarias para su formación. La coevaluación refuerza la asimilación de los contenidos de las materias, así como la capacidad crítica y autocrítica.

Específicamente, el compartir la evaluación del profesorado con la utilización de la metodología de evaluación interpares, tanto en su modalidad de evaluación entre grupos como individual, permite desarrollar competencias incluidas en el Libro Blanco para Grado en Economía de la Agencia Nacional de Evaluación de la Calidad y Acreditación (ANECA) como la capacidad crítica y autocrítica; así como la implicación del alumnado en las clases.

En el caso de la materia Econometría II, la evaluación entre grupos reforzó el carácter colaborativo de la actividad al tener que consensuar entre los miembros de cada equipo la asignación de valoraciones, fomentando la negociación y las habilidades interpersonales. Al mismo tiempo la evaluación a sus 
compañeros y compañeras proporcionó al estudiantado una opinión más realista de sus logros y dificultades y una guía en su proceso de aprendizaje, que le permitió aprender más y mejor.

En el caso de la materia Política Económica I, la modalidad de evaluación individual reforzó el grado de aprovechamiento que reportó al alumnado no expositor su participación en cada sesión de trabajo. Ante la responsabilidad de ejercer como calificadores individuales dedicaron más atención a los contenidos presentados y a las actuaciones de diferentes estudiantes; desempeñando roles de mayor relevancia que de pasivos oyentes.

Cabe destacar que ambas experiencias tuvieron una valoración positiva por parte del alumnado. Entre los aspectos más valorados se encuentran el aumento de su grado de implicación en la materia y la mejora de su capacidad autoevaluadora.

La experiencia de evaluación realizada nos confirma que la coevaluación es un procedimiento muy útil al proceso docente, en particular por las contribuciones del proceso de coevaluación a la calidad del aprendizaje.

\section{Referencias}

Aguayo, E. (2010) Aprendizaje acumulativo y aplicado con clases interactivas: el caso de Econometría I en la USC, RIDU: Revista d'Innovació Docent Universitària, 2, pp. 40-50.

Agencia Nacional de Evaluación de la Calidad y Acreditación (ANECA) (2005) Libro Blanco. Título de Grado en Economía y en Empresa. En: http://www.aneca.es/var/media/150292/libroblanco_economia_def.pdf

Álvarez Valdivia, I. (2008) La coevaluación como alternativa para mejorar la calidad del aprendizaje de los estudiantes universitarios: valoración de una experiencia. Revista Interuniversitaria de Formación del Profesorado, 22(3), pp. 127-140.

Dochy, F., Segers, M. y Dierick, S. (2002) Nuevas Vías de Aprendizaje y Enseñanza y sus Consecuencias: una Nueva Era de Evaluación. Boletín de la Red Estatal de Docencia Universitaria, 2(2), pp. 13-31.

Dochy, F., Segers, M. y Sluijsmans, D. (1999) The use of self-, peer and co-assessment in higher education: A review. Studies in Higher education, 24(3), pp. 331-350.

Falshikov, N. (1986) Product comparisons and process benefits of collaborative peer group and self assessment. Assessment and Higher Education, 11, pp. 146-166.

Falchikov, N. (2001) Learning together: peer tutoring in higher education. Routledge Falmer. London.

Gessa, A. (2011) La coevaluación como metodología complementaria de la evaluación del aprendizaje. Análisis y reflexión en las aulas universitarias. Revista de Educación, 354, pp. 749-764.

Gómez Ruiz, M.Á. y Quesada Serra, V. (2017) Coevaluación o evaluación compartida en el contexto universitario: La percepción del alumnado de primer curso. RIEE. Revista Iberoamericana de Evaluación Educativa, 10(2), pp. 930.

Hacker, D.J., Bol, L., Horgan, D.D. y Rakow, E.A. (2000) Test prediction and performance in a classroom context. Journal of Educational Psychology, 92(1), pp. 160-170.

Hall, K. (1995) Co-assessment: participation of students with staff in the assessment process. A report of Work in Progress. 2nd European Electronic Conference On Assessment And Evaluation, EARLI-AE list European Academic \& Research Network (EARN). En: http://listserv.surfnet.nl/archives/earli-ae.html. 
Ibarra, M.S.; Rodríguez, G. y Gómez, M.A. (2012) La evaluación entre iguales: beneficios y estrategias para su práctica en la universidad. Ministerio de Educación.

Prins, F., Sluijsmans, D.M., Kirschner, P.A. y Strijbos, J.W. (2005) Formative peer assessment in a CSCL environment: a case study. Assessment \& Evaluation in Higher Education, 30(4), pp.417-444. En: http://hdl.handle.net/1820/916.

Sánchez, M.P. (coord.) (2010) Técnicas docentes y sistemas de evaluación en Educación Superior. Narcea S.A. Editorial, Madrid.

Somervell, H. (1993) Issues in Assessment, Enterprise and Higher Education: The Case for Self-, Peer and Collaborative Assessment. Assessment and Evaluation in Higher Education, 18(3), pp. 221-33.

Topping, K. (1998) Peer assessment between students in colleges and universities. Review of Educational Research, 68(3), pp. 249-276.

Universidade de Santiago de Compostela (2009) Plan de Estudios del Grado de Economía. En: http://www.usc.es/gl/centros/ecoade/modules/news/news_0002.html.

Zambrana, P. (2010) La adquisición de competencias en el marco de la enseñanza de la Historia del Derecho: la preparación y exposición de temas por los alumnos como técnica docente. Revista Jurídica de Investigación e Innovación Educativa, 1, pp. 77-94. En: http://www.eumed.net/rev/rejie. 